$\mathbb{T}$ periodica polytechnica

Transportation Engineering

$36 / 1-2(2008) 2123$

doi: 10.3311/pp.tr.2008-1-2.04

web: http://www.pp.bme.hu/tr

(c) Periodica Polytechnica 2008

RESEARCH ARTICLE

\section{A simple method to reduce scale effect on wake and thrust deduction fractions of ships}

Zoltán Benedek

Received 2007-03-03

\begin{abstract}
The author propose a simple method for the calculation of the necessary thrust and the open water velocity of propeller from the results of model experiments According to the investigation of the measured results of several geosim it may be stated, that it is a better approximation to calculate the thrust and the open water velocity by means of the proposed method instead of thrust deduction and wake fraction.
\end{abstract}

\section{Keywords}

Scale effect $\cdot$ wake fraction $\cdot$ thrust deduction fraction
Zoltán Benedek

Department of Aeroplans and Ships, BME, H-1111 Budapest Sztoczek u. 6., Hungary

e-mail: benedek@rht.bme.hu

\section{Introduction}

Several investigations of model families verified, that the effective wake and the thrust deduction fractions of the models made in different scale are different [1-3]. For example these fractions of the Victory model family at 16 knots corresponding ship speed

$\begin{array}{lcccccc}\text { Modelscale } & 6 & 18 & 23 & 30 & 40 & 50 \\ 10^{3} . w & 251 & 324 & 346 & 348 & 367 & 403 \\ 10^{3} . t & 268 & 217 & 227 & 224 & 201 & 194\end{array}$

The advance velocity of propeller in open water condition of the model made in scale of 6 calculated by the wake fraction of the model made in 50 model scale is $2.01 \mathrm{~m} / \mathrm{s}$ and the real value is $2.56 \mathrm{~m} / \mathrm{s}$ determined by the measured results of the model made in scale of 6 . The difference is $0.55 \mathrm{~m} / \mathrm{s}, 22 \%$.

The propeller thrust of model made in scale of 6 calculated by means of the thrust deduction fraction of the model made in scale of 50 is $2371 \mathrm{~N}$ and the real value is $2663 \mathrm{~N}$ determined by measured results of the model made in scale of 6 . The difference is $262 \mathrm{~N}, 10 \%$.

\section{The Proposed Method}

The necessary thrust have to be equal to the resistance of the propelled ship. The main part of difference of the resistances of the ship in self propelled condition, and in towed condition is caused by change of pressure near the aft part of ship body. The difference of the frictional resistance is negligible. According to this we may use the same method which is proposed by William Froude for the conversion of the model resistance:

$$
T=\left[\frac{T_{m}}{0.5 \rho v_{m}^{2} S_{m}}-c_{f m}+c_{f}\right] 0.5 \rho v^{2} S
$$

$\begin{array}{cl}\text { where } & \\ v & \text { ship speed } \\ v_{m} & \text { corresponding model speed } \\ T & \text { thrust of ship } \\ T_{m} & \text { thrust of model } \\ \rho & \text { density of water }\end{array}$


$S \quad$ wetted surface of ship

$S_{m} \quad$ wetted surface of model

$c_{f} \quad$ frictional resistance coefficient of ship

$c_{f m}$ frictional resistance coefficient of model

The measured results of model families prove that the proposed method is not only simpler, but gives better results as the calculation by means of thrust deduction fraction (Tables I. IV.).

The advance velocity of propeller in open water condition and the ship speed are different because of there is an energy loss of water before of propeller in the behind condition of propeller,

In open water condition the energy given to $1 \mathrm{~kg}$ water by the propeller is

$$
e=\frac{v_{R}^{2}}{2}-\frac{v_{A}^{2}}{2}
$$

and in behind condition

$$
e=\frac{v_{R}}{2}-\frac{v^{2}}{2}+c \cdot \frac{v_{O}^{2}}{2}
$$

where

$v \quad$ ship speed

$v_{A} \quad$ advance velocity of propeller in open water condition

$v_{O} \quad$ axial velocity of water at the propeller

$v_{O}=\frac{v_{A}+\sqrt{v_{A}^{2}-\frac{2 T}{\rho A}}}{2} \quad A=\frac{D^{2} \cdot \pi}{4}$

$T \quad$ thrust of propeller

$v_{R} \quad$ the maximal velocity of water behind the propeller

c loss coefficient

In the two conditions the energy given by the propeller are the same, when the thrust and the revolution of propeller are the same,

$$
\frac{v_{R}^{2}}{2}-\frac{v_{A}^{2}}{2}=\frac{v_{R}}{2}-\frac{v^{2}}{2}+c \cdot \frac{v_{O}^{2}}{2}
$$

From this the loss coefficient in the behind condition is

$$
c=\frac{v^{2}-v_{A}^{2}}{v_{O}^{2}}
$$

It may be assumed, that the most part of losses are caused by the friction. Therefore the calculated values of loss coefficient of model $c_{m}$ multiplied by the ratio of the frictional resistance coefficient of the model and ship

$$
c=c_{m} \frac{c_{f}}{c_{f m}}
$$

we get the energy loss fraction of the ship. By means of this the open water velocity of the popeller of the ship can be calculate as follows:

$$
v_{A}=\sqrt{v^{2}-c \cdot v_{O}^{2}}
$$

\section{Results}

According to the investigated measurement results of several geosim it may be stated, that it is a better approximation for calculating the open water velocity by means of this $c$ loss coefficient instead of wake fraction. In tables I. - IV. there are necessary thrusts an open water velocities of the investigated geosims. In the first lines are the real values of thrust of the largest model, according to the model experiments. In the second lines are the values calculated by means of the the thrust deduction fraction of the smallest model. In the third lines the calculated values from the thrust of the smallest model by means of the proposed method. In the fourth lines are the real values of open water velocities of propeller of the largest model, according to the model experiments. In the fifth lines are the values calculated by means of the wake fraction of the smallest model. In the sixth lines the values calculated by the $c_{m}$ loss coefficient of the smallest model. The frictional resistance coefficients are calculated by the length and speed of models according to the ITTC 1957 formula.

\section{Conclusion}

According to the investigation of the measured results of several geosims it may be stated, that it is a better approximation to calculate the necessary thrusts by means of the proposed method instead by means of the thrust deduction coefficient,and the open water velocity of propeller by means of the proposed $c$ loss coefficient instead of wake fraction.

\section{References}

1 Lap AJW, Van Manen JD, Scale Effect Experiments of Victory Ship and Models, Transaction of the Royal Institute of Naval Architects CIV (1962), 23-55.

2 Modelfamilienversuche für einen 20.000 tdw Tanker., Bericht der Versuchsanstalt Für Wasserbau und Schiffbau (1964). (Bericht 280/64).

3 Modelfamilienversuche für das Forschungschiff Meteor, Bericht der Versuchsanstalt Für Wasserbau und Schiffbau (1965). (Bericht 321/65).

4 Grothues-Spork H, On Geosim Tests for the Research Vessel "Meteor" and a Tanker, Transactions of the Institute of Marine Engineers 77 (Oct. 1965), no. 10.

5 Benedek Z, An Investigation of the Scale Effect on Self-propulsion Factors, International Shipbuilding Progress 15 (1968), 78-95. 
Tab. 1. Victory modelfamily(at even keel). Scale of largest model: 6. Scale of smalles model:50.
Tab. 2. Victory modelfamily (trimmed by stern). Scale of largest model:6. Scale of smalles model:50

\begin{tabular}{lcccc}
\hline Shipspeed in knotes & 14 & 15 & 16 & 17 \\
\hline Meassured thrust & 1932.9 & 2250.6 & 2633.0 & 3106.7 \\
Thrust calculated by means of thrust deduction fraction & 1747.7 & 2018.2 & 2371.5 & 2900.0 \\
Thrust calculated by means of the proposed method & 1920.2 & 2210.2 & 2586.2 & 3162.2 \\
Measured value of the open- water velocity of propeller & 2.18 & 2.34 & 2.52 & 2.69 \\
Calculated velocity by means of wake fraction & 1.78 & 1.89 & 2.01 & 2.13 \\
Calculated velocity by means of proposed method & 2.23 & 2.38 & 2.53 & 2.68 \\
\hline
\end{tabular}

\begin{tabular}{lcccc}
\hline Shipspeed in knotes & 14 & 15 & 16 & 17 \\
\hline Meassured thrust & 1696.7 & 1863.3 & 2286.9 & 2691.9 \\
Thrust calculated by means of thrust deduction fraction & 1551.4 & 1793.6 & 2069.7 & 2438.7 \\
Thrust calculated by means of the proposed method & 1719.7 & 1994.2 & 2287.3 & 2672.9 \\
Measured value of the open- water velocity of propeller & 2.10 & 2.27 & 2.42 & 2.54 \\
Calculated velocity by means of wake fraction & 1.68 & 1.80 & 1.91 & 2.03 \\
Calculated velocity by means of proposed method & 2.15 & 2.31 & 2.45 & 2.60 \\
\hline
\end{tabular}

Tab. 3. Strinda modelfamily. Scale of largest model: 25. Scale of smalles model: 55 .

Tab. 4. Meteor modelfamily. Scale of largest model: 13.75 . Scale of smalles model: 25 .

\begin{tabular}{lcccc}
\hline Shipspeed in knotes & 12 & 14 & 16 & 18 \\
\hline Meassured thrust & 30.69 & 43.25 & 60.80 & 86.79 \\
Thrust calculated by means of thrust deduction fraction & 29.66 & 42.00 & 58.96 & 111.32 \\
Thrust calculated by means of the proposed method & 31.78 & 44.80 & 63.69 & 91.80 \\
Measured value of the open- water velocity of propeller & 0.81 & 0.95 & 1.09 & 1.22 \\
Calculated velocity by means of wake fraction & 0.66 & 0.83 & 1.11 & 1.11 \\
Calculated velocity by means of proposed method & 0.77 & 0.94 & 1.10 & 1.25 \\
\hline
\end{tabular}

\begin{tabular}{lcccc}
\hline Shipspeed in knotes & 8 & 10 & 12 & 14 \\
\hline Meassured thrust & 12.75 & 22.56 & 35.30 & 57.37 \\
Thrust calculated by means of thrust deduction fraction & 11.10 & 18.25 & 29.89 & 68.70 \\
Thrust calculated by means of the proposed method & 12.34 & 20.06 & 31.74 & 70.35 \\
Measured value of the open- water velocity of propeller & 0.81 & 1.01 & 1.21 & 1.41 \\
Calculated velocity by means of wake fraction & 0.68 & 0.92 & 1.15 & 1.36 \\
Calculated velocity by means of proposed method & 0.74 & 0.99 & 1.22 & 1.45 \\
\hline
\end{tabular}

